\title{
Super-Resolution Light Microscopy Using Plasmonic Gratings
}

\section{Aaron Wood, ${ }^{1}$ Biyan Chen, ${ }^{2}$ Joseph Mathai, ${ }^{2}$ Sangho Bok, ${ }^{2}$ Sheila Grant, ${ }^{1}$ Keshab Gangopadhyay, ${ }^{2}$ Peter V. Cornish, ${ }^{3}$ and Shubhra Gangopadhyay ${ }^{2 *}$}

${ }^{1}$ Department of Bioengineering, 139 and 141A Engineering Building West, University of Missouri, Columbia, MO 65211

${ }^{2}$ Department of Electrical and Computer Engineering, 139 and 141A Engineering Building West, University of Missouri,

Columbia, MO 65211

${ }^{3}$ Department of Biochemistry, 117 Schweitzer Hall, University of Missouri, Columbia, MO 65211

*gangopadhyays@missouri.edu

\begin{abstract}
A novel platform for super-resolution imaging has been devised that employs plasmonic gratings fabricated using glancing angle deposition (GLAD) of silver. GLAD was found to produce a large population of unique nanostructures over the entire plasmonic grating. These nanostructures excite nearby fluorescent molecules to improve spatial resolution to sub-diffraction limit distances while also increasing signal-to-noise ratio (SNR). For example, the improved localization precision produces $65 \mathrm{~nm}$ image resolution on a highly concentrated fluorescent sample. These inexpensive plasmonic GLAD gratings have potential to improve fluorescent intensity and resolution over a wide range of applications.
\end{abstract}

\section{Introduction}

Super-resolution microscopy, where the resolution can break the diffraction limit, plays a critical role in advanced biological research. Super-resolution microscopy enables scientists to observe the movement and interaction of individual molecules and nanoscale features [1-6]. Several super-resolution techniques have been developed over the past few years that have led to a better understanding of biological systems. These include near-field scanning optical microscopy (NSOM) [7-12], far-field superlens [13], photo-activated localization microscopy (PALM) $[14,15]$, and stochastic optical reconstruction microscopy (STORM) $[16,17]$. These techniques typically use mathematical models and a series of diffraction-limited images or complicated optical, mechanical, and electrical setups to increase the spatial resolution to sub-diffraction limit levels.

However, there are still limitations to certain superresolution techniques. For example, tip-based techniques (such as NSOM) require lengthy scan times when imaging large sample areas because the tip must be rastered over the entire surface [12, 18]. Unlike tip/probe-based techniques, localization microscopy (PALM and STORM) pinpoints the centroids of well-separated single molecules using hundreds or thousands of images to reconstruct a few super-resolution images. The location precision can be greatly increased by combining this technique with microscopes equipped with optical sectioning capabilities, such as total internal reflection fluorescence microscopy (TIRFM), which enhances the signal-to-noise ratio (SNR) [3].

In this article we describe a novel fluorescence enhancement platform, recently published in Nanoscale [19], that further enhances the SNR without the need for complex optical setups. This platform uses a plasmonic grating with unique nanostructures grown on the surface. Plasmonic gratings, that is, ridge-groove structures of a noble metal such as gold or silver, are able to generate intense electromagnetic fields (EM fields) when light at specific wavelengths shines onto the grating surface at specific angles. This phenomenon is known as surface plasmon resonance (SPR). After growing additional nanostructures on the grating, we found that these nanostructures can concentrate the EM field generated by the plasmonic grating to greatly enhance the number of photons emitted by nearby fluorescent molecules. When compared with STORM/ PALM, the enhanced SNR of the plasmonic grating enables single-molecule localization precision over a wide range of fluorophore concentrations using a simple epifluorescence microscope, eliminating the need for TIRFM. Additionally, plasmonic gratings have an inherently wider coupling angle range than prism-based plasmonics, which eliminates the need for laser excitation with fine-angle control and allows users to excite the grating with the illumination from a typical microscope objective.

\section{Materials and Methods}

Grating microstructure. Plasmonic gratings are relatively inexpensive to produce compared to those produced by electronbeam lithography and may be fabricated from the grating structures found on high-definition DVDs or Blu-ray discs. After producing the grating profile in polymethylsilsesquioxane (PMSSQ) on a microscope slide using microcontact lithography, the gratings are coated with a thin film of silver metal. The latter process is accomplished via GLAD where the silver is deposited at an angle relative to the grating surface [20-25]. In previous publications, we developed normally deposited silver plasmonic gratings with random nanogaps to enhance single molecule fluorescence [20] and to detect DNA oscillation [21]. While a conformally deposited silver film provides only some of the plasmonic properties needed, the GLAD process forms a silver film with an abundance of different nanostructures such as nanoprotrusions and nanogaps, as seen in Figures $1 \mathrm{a}$ and $1 \mathrm{~b}$ [19].

Nanoprotrusions are protruding silver grains forming on top of the grating ridges that can generate "hotspots" via localized surface plasmon resonance (LSPR) that excites nearby fluorophores. Nanogaps are narrow crevices that form at the base of the grating ridge in the shadowed region where the flux of silver was greatly reduced. During the development of these GLAD gratings, it was found that a deposition angle with respect to the surface of $\alpha=60^{\circ}$ produced the highest density of these two nanostructures. This is advantageous because a high density of plasmonic hotspots enables instantaneous imaging over a large area instead of the point-by-point scanning used in NSOM and provides a better SNR $(\sim 28)$ than more complicated and expensive systems (NSOM SNR 7) [26] and much higher than the minimum SNR for single molecule imaging ( 3) [27]. 


\section{June 4-9,}

\section{LEHIGH}

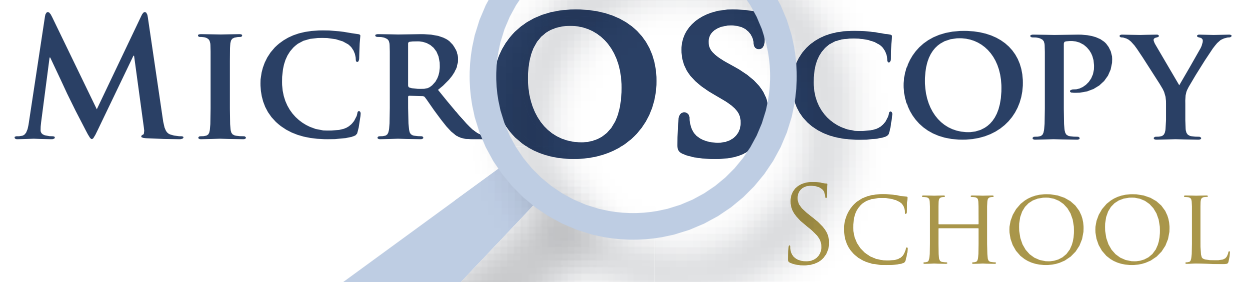

Lehigh University, Bethlehem, PA USA

\section{MAIN COURSES}

SCANNING ELECTRON MICROSCOPY AND X-RAY MICROANALYSIS

June 5-9

\section{SPECIALIZED COURSES}

FOCUSED ION BEAM (FIB): Instrumentation and Applications June 5-9

PROBLEM SOLVING:

Interpretation and Analysis of SEM/EDS/EBSD Data

June 5-9
INTRODUCTION TO SEM AND EDS FOR THE NEW OPERATOR June 4

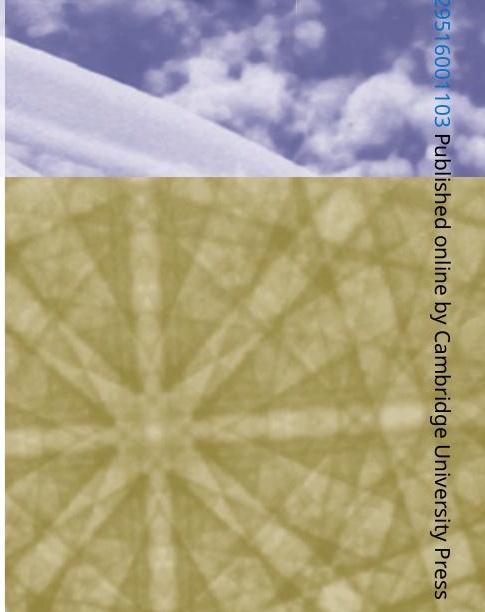




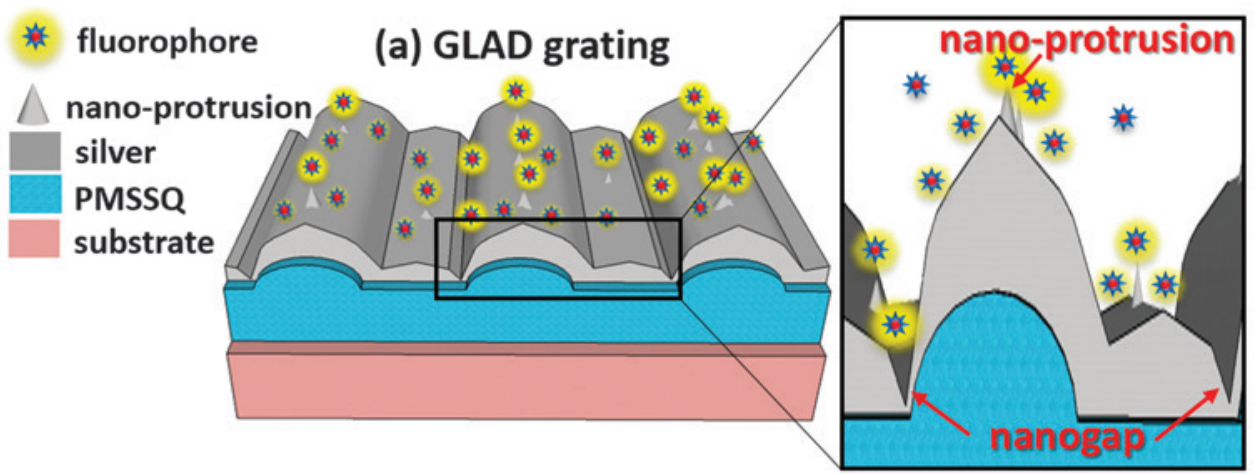

\section{(b) GLAD grating $\left(\alpha=60^{\circ}\right)$ Nano-protrusion}

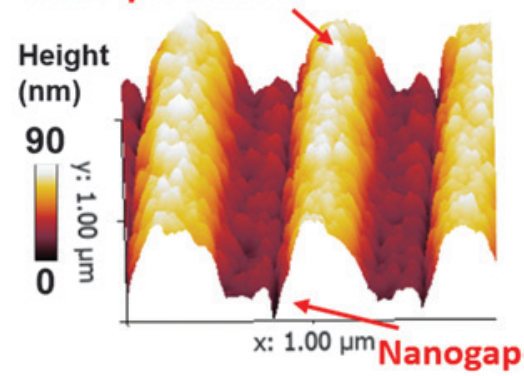

Figure 1: (a) Schematic of a silver plasmonic GLAD grating on a fluorescence-based application and an expanded schematic of the GLAD morphology with nanoprotrusions and nanogaps. The brighter yellow color of the image indicates higher fluorescent emission intensity. (b) AFM 3D contour map of silver GLAD grating rapidly deposited at an oblique deposition angle $\left(\alpha=60^{\circ}\right)$, exhibiting controlled nanogaps and nanoprotrusions (hotspots). Reproduced from [19] with permission, copyright 2016 RSC.

Grating coatings. The microcontact lithography process for making PMSSQ gratings from profiles stored on DVDs is described in detail in our previous publications $[20-25,28]$. This process can be used to fabricate PMSSQ gratings on both microscope slides and silicon wafers. Afterward, $2 \mathrm{~nm}$ thick chromium and $40 \mathrm{~nm}$ thick silver films were deposited onto the PMSSQ grating using thermal evaporation and a custom-built variable angle deposition stage that rotates the sample surface such that the metal flux is incident at $\alpha=60^{\circ}$ above the surface. During our study of the GLAD technique, we found that the shape, distribution, and size of the nanoprotrusions and nanogaps can be further modified by tuning the PMSSQ surface energy, incidence angle of the metal vapor, and the deposition rate. The platform can also be coated with different materials to improve its functionality for different applications. For example, the silver can be capped with silica $\left(\mathrm{SiO}_{2}\right)$ or gold to provide a binding site for biological studies. The GLAD grating can also be coated with a PMSSQ matrix that incorporates a dye for fluorescence studies.

FDTD simulations. Based on finite difference time domain (FDTD) simulations of electric-field distribution (Figure 2), the E-field is highly concentrated into hotspots located on both nanoprotrusions and nanogaps. Because the E-field is confined in both the axial and lateral directions, we can obtain better imaging resolution in both directions. It is important to note that the fluorescence enhancement obtained by a GLAD grating is proportional to the product of both the excitation and emission E-field enhancement [20]. The wide coupling wavelength and angle ranges of the GLAD gratings are well suited to enhance the fluorescence emission intensity. When this enhancement is combined with the extremely low background intensity due to the inherent absorptive nature of the plasmonic gratings, the resulting samples have an improved SNR. This greater SNR enables imaging of single molecules over a wide range of fluorophore concentration and improves the localization precision of super-resolution studies.

Single-molecule imaging. Typically, single-molecule imaging in the low fluorophore concentration range requires amplified fluorescence emission intensity and/or increased camera sensitivity such that the signal of a single fluorophore is distinguishable over background noise. On the other hand, this type of imaging in high fluorophore concentrations

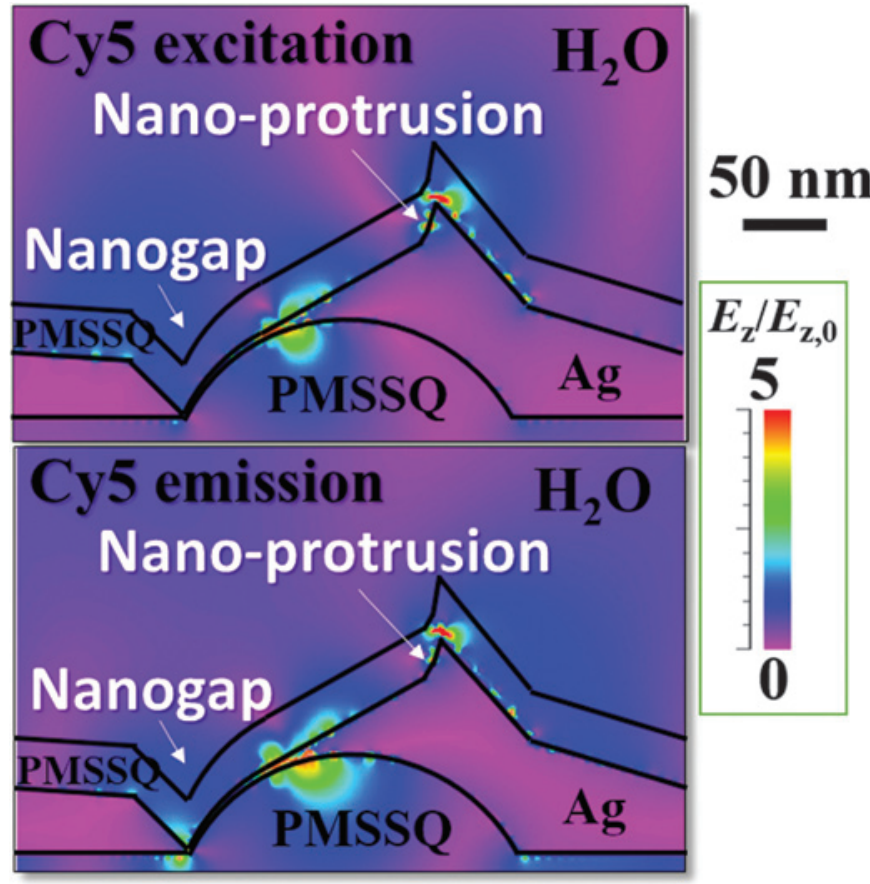

Figure 2: Simulated E-field (|Ez/Ez,0|) distribution for $40 \mathrm{~nm}$ thick silver GLAD gratings coated with $30 \mathrm{~nm} \mathrm{PMSSQ}$ in water environment at Cy5 excitation $\left(\lambda=642 \mathrm{~nm}, \theta=0^{\circ}\right)$ and emission $\left(\lambda=670 \mathrm{~nm}, \theta=12.2^{\circ}\right)$. Reproduced from [19] with permission, copyright 2016 RSC.

requires that the excitation be confined to a small volume and imaged without bulk averaging. The spatial resolution is then determined by the volume of the confined light in a plasmon-related nanostructure, which can be quite small when a nanoprotrusion or nanogap is less than $100 \mathrm{~nm}$ in width [29]. We tested the single-molecule imaging capabilities of these GLAD gratings by imaging samples containing several Cy5 fluorophore concentrations including the following: a surface-immobilized DNA/RNA duplex labeled with Cyanine-3 (Cy3) and Cyanine-5 (Cy5) (Figure $3 a$ and $3 b$ ) and spin-coated PMSSQ films (30 nm thick) laced with a lowand high-concentration range of Cy5 (Figure $3 \mathrm{c}$ and $3 \mathrm{~d}$ ). The fluorescence images and videos were taken with either an Andor iXon+ EMCCD camera or an ORCAFlash 2.8 CMOS camera with a $1 \times$ or $2 \times$ magnification collar. Because of the inherently 


\section{Cy5 concentration}
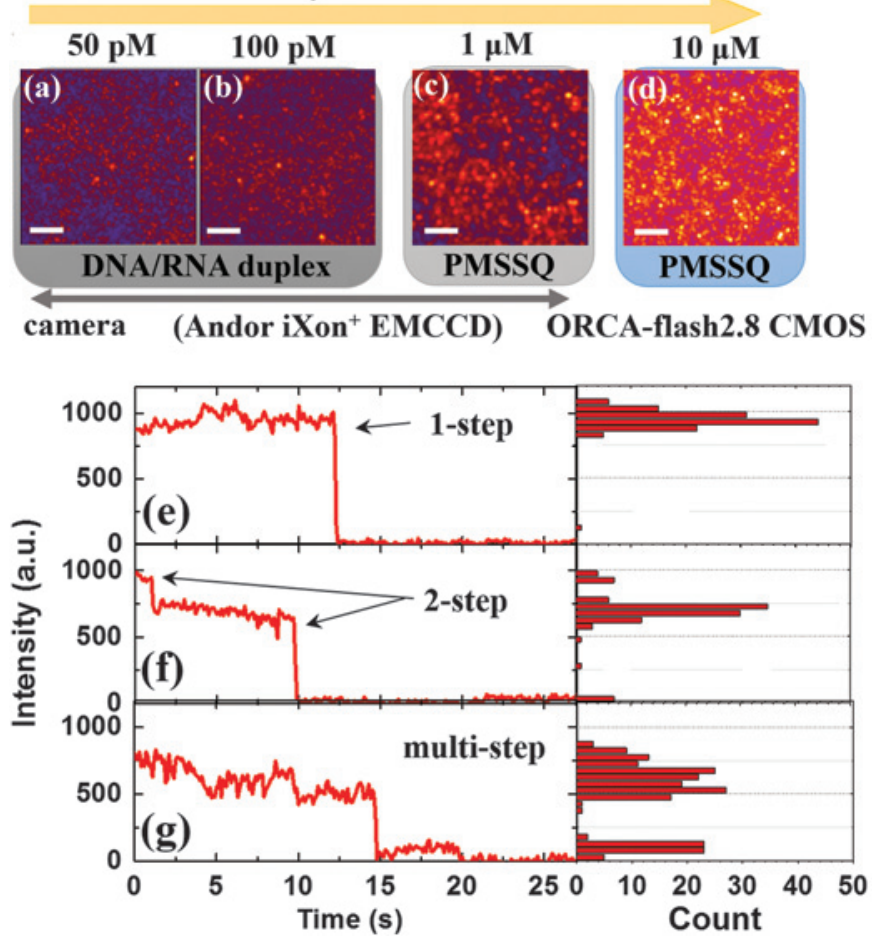

Figure 3: A wide range of dye concentrations were imaged using these silver GLAD gratings $\left(\alpha=60^{\circ}\right)$ : (a) $50 \mathrm{pM}$ and (b) $100 \mathrm{pM}$ DNA/RNA duplex-labeled with Cy3/Cy5; (c) $1 \mu \mathrm{M}$ and (d) $10 \mu \mathrm{M}$ Cy5 in PMSSQ matrix. Scale bar $=5 \mu \mathrm{m}$ for all images. (e-g) Representative time traces for $1 \mu \mathrm{M}$ Cy5 at hotspots with single/ double/multiple step bleaching behavior using $550 \mu \mathrm{W} 642 \mathrm{~nm}$ laser taken with $60 \times$ objective in epifluorescence mode. Reproduced from [19] with permission, copyright 2016 RSC.

large number of molecules and overlapped signals observed on highly concentrated fluorescent samples, we recorded the single/ double/multiple step photobleaching behavior on hotspots. The quantized photobleaching of each Cy5 molecule with a single "on" and "off" state enables the counting of dye molecules with relative ease by using the number of photobleaching steps, seen as distinct intensity plateaus, to estimate the number of molecules in each time trace [30-32]. Figures 3e-3g illustrate this counting technique with time traces extracted from the sample imaged in Figure 3c.
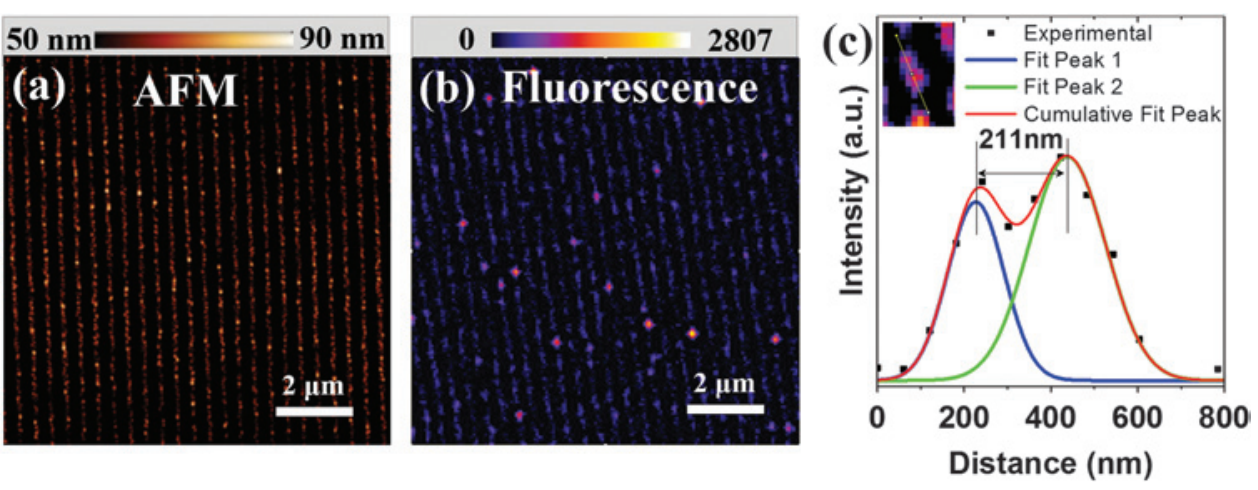

Figure 4: Comparison of hotspot density between (a) AFM image with the height range (50-90 nm) of the protruding grains highlighted and (b) false-colored fluorescence image of $10 \mu \mathrm{M}$ Rhodamine 6G dye in a PMSSQ matrix using a $60 \times$ WI objective (NA = 1.2) after background subtraction. (c) Intensity vs. distance plot from fluorescence image; inset shows two analyzed hotspots and the cross section used for profile analysis. The fluorescence images were taken by an ORCA-Flash 2.8 CMOS camera. Reproduced from [19] with permission, copyright 2016 RSC.

\section{Results}

Beating the diffraction limit. During our localization microscopy analysis, we observed a strong correlation between the density of hotspots in the fluorescent images and the density of nanoprotrusions observed in atomic force microscope (AFM) scans of the GLAD surface. These data support the idea that nanoprotrusions (Figure 4a) are capable of concentrating the E-field as seen in FDTD simulations, which results in the formation of the fluorescence hotspots (Figure 4b). Using single-molecule analysis of the fluorescence images, we observed two hotspots with a separation distance of $211 \mathrm{~nm}$ (Figure 4c). This distance would be too short to measure in a diffraction-limited image, but resolution obtained here was made possible by the far-field superlensing (FSL) effect of the GLAD gratings.

Localization microscopy. Despite the sub-diffraction limit resolution of the raw fluorescence images, they still cannot provide the exact size of nanostructures less than $\sim 210 \mathrm{~nm}$ without additional processing. This resolution limit is thought to arise from an overlap of the diffraction-limited, isotropic emission of some single molecules at the 0 -order and the sub-diffraction limit \pm 1 -order diffraction from surface plasmon-coupled emission (SPCE). To further improve the resolution, we have used localization microscopy concepts to analyze the time-trace videos from the sample in Figure 3c. In localization microscopy, the coordinates of temporally separated single molecules are found via a fitted, isolated point-spread function (PSF). While PSF fitting is used by many current imaging techniques, those methods often require well-spaced single molecules to obtain accurate sample information [33]. This becomes a much larger limitation when applied to imaging fluorescently labeled biological samples where the spatial density of fluorophores is such that they are too overlapped for single-molecule resolution. The advantage of the GLAD gratings is that the high SNR (28 \pm 9 ) provided by the nanoprotrusions and nanogaps allows us to better resolve individual molecules even when the signals of several molecules are overlapped. This in turn allows us to obtain a well-fitted PSF at concentrations beyond the capabilities of other systems.

In addition to PSF analysis we also employed the multipleemitter fitting analysis (MFA), which is capable of processing a much higher density of emitters than with single-emitter analysis. MFA uses the maximum likelihood estimator to obtain position estimates of many single-molecule emissions over several images and combines them into a single image (Figure 5). This results in an order of magnitude improvement in the tolerance of the analysis routine with respect to the single-frame active emitter density [34]. We tested the capabilities of MFA analysis using the sample in Figure $3 \mathrm{c}$ and compared the images to the original fluorescence images. We found that the resolution of the superresolution reconstructed fluorescence 

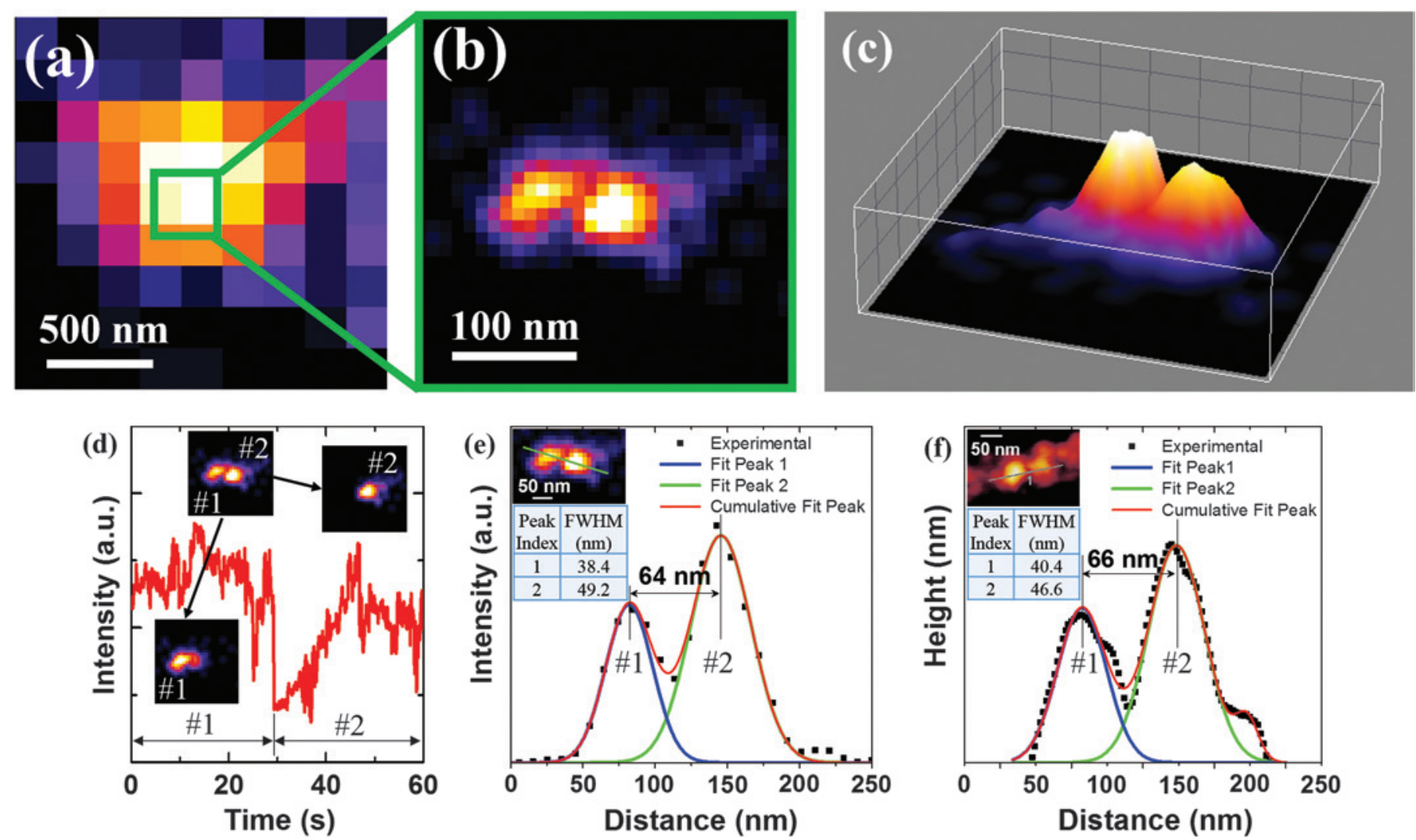

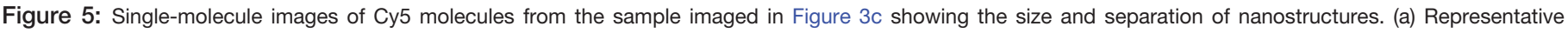

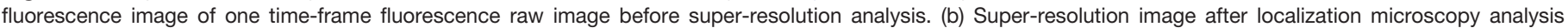

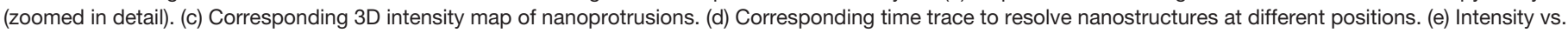

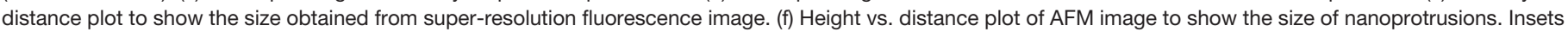

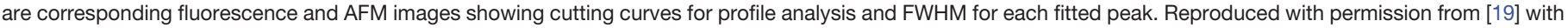
permission, copyright 2016 RSC.

image had been significantly improved. The minimum separation distance was reduced to $\sim 65 \mathrm{~nm}$, and the full width at half maximum (FWHM) of the emission was reduced to $\sim 40 \mathrm{~nm}$ (Figure 5e). This separation distance and FWHM correlate well with separation and size of nanoprotrusions measured with AFM at several locations (Figure 5f). Due to the presence of a conformal PMSSQ coating with embedded dye molecules, we could not obtain surface information with AFM after fluorescence imaging, but we are currently developing methods to track specific molecules in both fluorescence and AFM.

\section{Discussion}

We have developed a plasmonic grating platform that can simply and effectively extend the resolution limits of any fluorescence microscope by behaving as a far-field superlens (FSL). An FSL operates by enhancing the evanescent high spatial frequency information at the surface and converting it into propagating information that is detectable in the far-field. Super-resolution imaging using a metallic grating as an FSL has also been accomplished by other research groups but with brightfield imaging rather than fluorescence imaging $[12,35,36]$. Super-resolution sample information under bright-field conditions can be obtained using silver windows to block the 0 -orderdiffractedlightcontaining diffraction-limited information and collecting the \pm 1 -order diffraction containing the super-resolution information [7]. However, in fluorescence imaging, SPCE is used to obtain super-resolution information. The SPCE effect occurs when an excited fluorophore within $100 \mathrm{~nm}$ from the grating surface non-radiatively transfers energy back to the plasmonic grating as a radiative surface plasmon, which is then emitted as a photon at the SPR coupling angle [37]. In the case of fluorescence imaging, the excitation background light reflected from the sample is cut off by the fluorescence filter cube, but fluorescence emitted via SPCE at the \pm 1 diffraction order is free to pass through the filter cube. The reflected excitation light can be further reduced using an excitation polarizer with a randomly polarized light source, such as a halogen or xenon arc lamp. Based on the SPR dispersion relation, p-polarized light is the only polarization that can excite oscillations at the grating/dielectric interface, also known as surface plasmon polaritons. The s-polarized light is inherently unable to generate surface plasmons because the electric component of the EM wave is parallel to the grating and dielectric interface. With use of an excitation polarizer, the s-polarized light that is typically reflected from the sample can be eliminated before reaching the sample.

Ultimately, the GLAD grating platform more specifically extends the resolution limits: (1) it converts incident light into intense electric fields at the surface that can be further concentrated at nanoprotrusions and nanogaps to create 
hotspots; (2) the high density of hotspots can simultaneously excite fluorescent molecules over the entire imaging plane; and (3) the high SNR of emission from fluorophores excited by hotspots enables much brighter images with finer resolution using a simple epifluorescence microscope than with much more complicated super-resolution systems. Ultimately, GLAD gratings represent enormous potential as an easy-to-use platform that enhances the image resolution to sub-diffraction levels and enhances the fluorescence emission intensity several orders of magnitude above glass slides.

\section{Conclusion}

The GLAD grating platform developed in this article enables users to beat the diffraction limit and obtain an image resolution of $\sim 210 \mathrm{~nm}$ before processing and a resolution of $\sim 65 \mathrm{~nm}$ after point-spread function deconvolution. We also obtained better fluorescent signal detection over a wide concentration range of fluorophores because of the high SNR of the single molecule images on the GLAD grating (SNR 28). The ease of use and resolution enhancements from the GLAD grating platform makes it the ideal choice for numerous applications in single-molecule fluorescence imaging.

\section{Acknowledgements}

Funding for this work was provided by a grant from the National Science Foundation (Award No. ECCS-1102070).

\section{References}

[1] CG Galbraith and JA Galbraith, J Cell Sci 124 (2011) 1607-11.

[2] DS Kim et al., Nano Lett 9 (2009) 3619-25.

[3] L Schermelleh et al., J Cell Biol 190 (2010) 165-75.

[4] G Shtengel et al., Proc Natl Acad Sci USA 106 (2009) 3125-30.

[5] Y Wang et al., Nano Lett 13 (2013) 5233-41.

[6] BYTD Harris et al., T. B. Laboratories and I. A. Room, 1994.

[7] Z Liu et al., Nano Lett 7 (2007) 403-08.

[8] Y Fu and X Zhou, Plasmonics 5 (2010) 287-310.

[9] Z Xie et al., Plasmonics 6 (2011) 565-80.
[10] X Zhang and Z Liu, Nat Mater 7 (2008) 435-41.

[11] Y Xiong et al., Nano Lett 7 (2007) 3360-65.

[12] S. Kawata et al., Nat Photonics 3 (2009) 388-94.

[13] JB Pendry, Phys Rev Lett 85 (2000) 3966-69.

[14] ST Hess et al., "Ultrahigh Resolution Imaging of Biomolecules by Fluorescence Photoactivation Localization Microscopy" in Micro and Nano Technologies in Bioanalysis: Methods in Molecular Biology, series vol. 544, eds. RS Foote and JW Lee, Springer, Clifton, NJ, 2009, p. 544, 483-522.

[15] E Betzig et al., Science 313 (2006) 1642-45.

[16] MJ Rust et al., Nat Methods 3 (2006) 793-95.

[17] M Bates et al., Cold Spring Harb Protoc 8 (2013) 498-520.

[18] Y Wang et al., Nano Lett 8 (2008) 3041-45.

[19] B Chen et al., Nanoscale 8 (2016) 12189-201.

[20] B Chen et al., Nanobiomedicine 2 (2015) 1.

[21] S Basuray et al., J Nanomedicine Nanotechnol 6 (2015) 1.

[22] K Bhatnagar et al., Nanotechnology 23 (2012) 495201.

[23] CM Darr et al., Sens Actuators B Chem 202 (2014) 1088-96.

[24] US 20140226207 A1, 2014.

[25] B Chen et al., ECS Trans 61 (2014) 69-82.

[26] X Michalet and S Weiss, Biophysics 3 (2002) 619-44.

[27] T Tanii et al., Phys Rev E 88 (2013) 012727.

[28] AJ Wood et al., RSC Adv 5 (2015) 78534-44.

[29] S Kawata et al., Nat Photonics 3 (2009) 388-94.

[30] P Dedecker et al., Opt Express 15 (2007) 3372.

[31] R Roy et al., Nat Methods 5 (2008) 507-16.

[32] Y Chen et al., Mol Biol Cell 25 (2014) 3630-42.

[33] E Tolstik et al., The Optics Encyclopedia, Wiley, Hoboken, NJ, 2015, 1-31.

[34] F Huang et al., Biomed Opt Express 2 (2011) 1377.

[35] H Lee et al., Solid State Commun 146 (2008) 202-07.

[36] S Durant et al., J Opt Soc Am B 23 (2006) 2383-92.

[37] JR Lakowicz et al., Biochem Biophys Res Commun 307 (2003) 435-39.

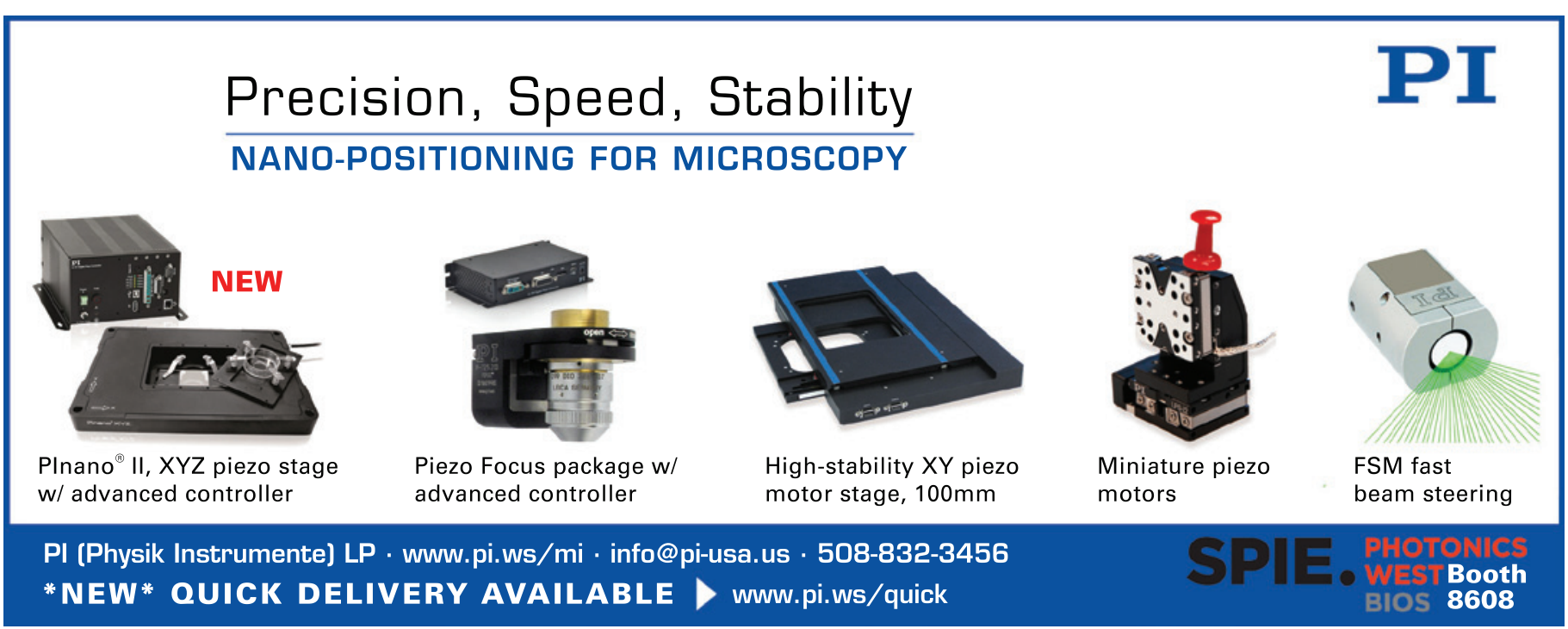

